\title{
Message Delivery and Relevant Simulation Tools for Vehicular Ad hoc Networks: A survey
}

\author{
Ahmed Ali ${ }^{1, a}$, Wang Dong ${ }^{1, b}$ and Li RenFa ${ }^{1, c}$ \\ ${ }^{1}$ College of Information Science and Engineering, Hunan University, Changsha 410082, China \\ aahmed_ali@hnu.edu.cn, bwangd@hnu.cn, cscc_Irf@hnu.cn
}

Keywords: Intelligent Transportation Systems, Message Query Telemetry Transfer, Simulation Tools, Vehicular Ad hoc Networks.

\begin{abstract}
Message Delivery among vehicular nodes is essential to achieve safe transportations. Due to the severe need for broadcasting safety messages over Vehicular Ad hoc Networks, several protocols have been proposed that target dominant problems including broadcast storm, hidden nodes and sparse connectivity. However, researchers still confused about suitable simulation tool selections. In this paper, we review state-of-art research in the field of vehicular networks. We introduce a collective view for work done concerning message delivery, challenges and future trends. Finally, we identify the most common simulation tools; their advantages and categorizations.
\end{abstract}

\section{Introduction}

Nowadays, Intelligent Transportation Systems (ITS) are considered the future of transportation [1], especially when Information Technology (IT) starts to knock transportation field. IT can magically transform concrete items such as traffic lights and vehicles to smart components. ITS includes different Vehicular Ad hoc Networks (VANETs) applications including traffic monitoring. VANETs aim to ensure safe roads by supporting ubiquitous connectivity among vehicular users. Traffic awareness can be ensured by periodically broadcasting real-time information among vehicles. Thus, Vehicle-to-Vehicle (V2V) and Vehicle-to-Roadside (V2R) communications are considered the backbone of VANETs [2].

In VANETs, Message Delivery (MD) protocols should achieve specified targets including short latency, high reliability, and robust connections. If drivers are given updated information regarding the road status, they will avoid dangerous situations or traffic jams. In this article, we survey popular MD protocols in VANETs and mention hot topics related to this research area. Unlike [3], we focus on protocols including both V2R and V2V communications. Moreover, we acquaint the VANETs simulation tools used to test various MD protocols.

This paper is organized as follows: In section 2, VANETs Architecture is briefly explained. Section 3 surveys the current MD protocols and their classifications. In section 4, we introduce the suitable VANETs simulations software and their classes. Finally, we conclude our survey and discuss challenges for future work manuscripts must be in English. Please keep a second copy of your manuscript in your office (just in case anything gets lost in the mail). When receiving the manuscript, we assume that the corresponding authors grant us the copyright to use the manuscript for the book or journal in question. Should authors use tables or figures from other Publications, they must ask the corresponding publishers to grant them the right to publish this material in their paper.

\section{VANETs Architecture}

The integration of wireless technologies in VANETs has fascinated researchers since 1980s due to IEEE 802.11 wide adoption [4]. VANETs are an application of Mobile Ad hoc Networks (MANETs), with a distinctive feature that is: "messages flow according to road topology and in a predetermined direction" [5]. Additionally, VANETs are characterized by the highly dynamic network topology. 
Although power constraint is not vital in VANETs, network lifetime is too short due to the unstable nature.

VANETs allow direct communications among vehicles and roadside units to exchange frequent messages. They operate on a special frequency band known as Dedicated Short-Range Communication (DSRC). Communication among vehicles and roadsides are obtained through a wireless medium called Wireless Access in Vehicular Environment (WAVE). This DSRC/WAVE assembly defined by IEEE 802.11p and IEEE 1609 meets the short latency requirements of VANETs [6]. The IEEE 802.11p standard usually operates on the 5.8 or $5.9 \mathrm{GHz}$ frequency bands, based on the regional authorities' regulations. In USA, $75 \mathrm{MHz}$ of the $5.9 \mathrm{GHz}$ frequency band is allocated to VANETs and the spectrum is divided into 7 channels (as illustrated in Fig. 1). They are classified into one Control Channel $(\mathrm{CCH})$ and 6 Service Channels (SCHs) with $10 \mathrm{MHz}$ frequency band for each channel plus $5 \mathrm{MHz}$ allocated for the guard band. Channel number is computed using Eq. 1:

$$
\mathrm{f}(\mathrm{CN})=5000+5 \mathrm{CN}(\mathrm{MHz}) \text {. }
$$

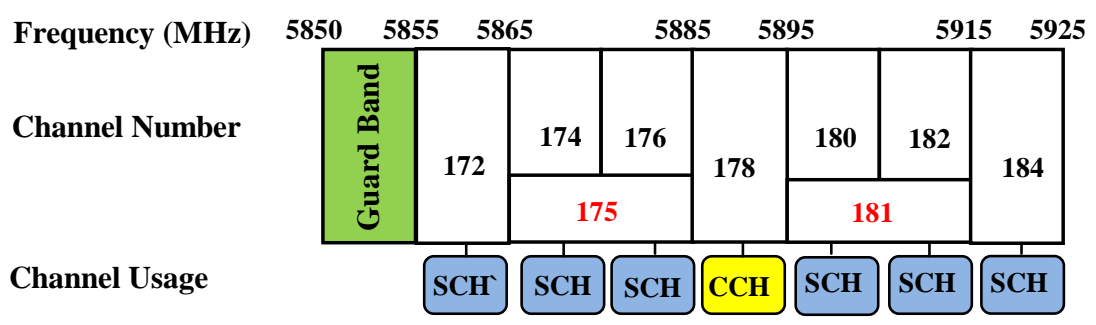

Fig. 1 DSRC Frequency Allocation in USA

Normally, VANETs contain three components including On-Board Unit (OBU), Application Unit (AU), and Roadside Unit (RSU). An OBU is mounted to each vehicle along with sensors to exchange information with other vehicles or RSUs. It is a WAVE device that consists of a user interface, a command processor, and an IEEE802.11p device in order to access DSRC spectrum. Also, a network device may be included for non-safety applications based on other wireless technologies such as IEEE $802.11 \mathrm{a} / \mathrm{b} / \mathrm{g} / \mathrm{n}$ [7]. Moreover, AUs are embedded in each OBU to run the provided applications.

On the other side, RSU is defined as an infrastructure which is placed in traffic lights, or bus stops. Similar to an OBU, It is equipped with a network device for DSRC communications. According to [8], it is shown that RSUs succeed to reduce network partition problems in VANETs and thus improve MD. Finally, an RSU provides OBUs with different services including information distribution, information source, and Internet access.

\section{Message Delivery Protocols}

The delivery of real-time information in VANETs can ensure accident-free transportation systems. However, vehicles high mobility and interrupted connections hinder the smooth flow of this information over the network. One popular form of MD in VANETs is the Publish/Subscribe messaging systems using Message Query Telemetry Transfer (MQTT) protocol [9]. It targets V2R communications by allowing information to be delivered based on its content. MQTT is an open protocol that ensures scalability and support dynamic application topologies as in VANETs [10].

Information Needed for Dissemination. Four types of information can be interchanged in VANETs according to the used application. DSRC is designed to give the highest priority to safety information. No matter wherever it is originated by an OBU or a RSU, it is necessarily concerning lives and possessions [11]. This information guides drivers to avoid dangerous situations such as high speed vehicle or bang brake. According to the US Department of Transportation and Vehicle Safety Communication Consortium, there are eight applications are introduced in Table 1 intended to use safety information [12]. 
According to the priority, traffic information comes in the second position after safety information. It helps drivers to select best routes by avoiding stuck into traffic jams. Recently, many commercial systems use the traffic information including GCMTravel [13]. Yet, vehicular users not only look for essential information but also infotainment information is needed. It is non-safety information such as electronic toll collection, advertisements, and parking areas [14]. In addition, content information is used for large size data (e.g. videos). These different types of information erect a challenging environment in VANETs.

Table 1 Applications of safety information type

\begin{tabular}{llccl}
\hline \multicolumn{1}{c}{ Application } & $\begin{array}{c}\text { Frequency } \\
{[\mathrm{Hz}]}\end{array}$ & $\begin{array}{c}\text { Maximum } \\
\text { Range [m] }\end{array}$ & Data Transmitted \\
\hline 1 & Curve Speed Warnings & 1 & 250 & - Curvature, Speed limit \\
2 & Traffic Signal Violation & 10 & 200 & - Signal status, Weather \\
3 & Sudden Brake & 10 & 200 & - Location, Velocity \\
4 & Pre-Crash Warning & 50 & 50 & - Type, Acceleration \\
5 & Left-Turn Assistance & 10 & 300 & - Road shape, Heading \\
6 & Lane Warning Change & 10 & 150 & - Acceleration, Turn sign \\
7 & Stop Sign Assistance & 10 & 300 & - Position, Velocity \\
8 & Collision Warning & 10 & 150 & - Vehicle type, Yaw rate \\
\hline
\end{tabular}

Message Delivery Protocols Categorization. The main goal of having efficient MD protocols is to serve the data needs of drivers and to reduce redundant data. They are used to exchange relevant message from source to destination in order to warn vehicles to avoid collisions or keep away from road congestion.

Vehicle-to-Vehicle Protocols. There are two types of MD protocols in V2V communications which are flooding and relaying protocols. In the first type, each vehicle participates in the broadcasting process. In these protocols, messages are sent without prior neighboring information which is not scalable high dense networks. They can easily cause the broadcast storm problem. Several solutions have been proposed to deal with this problem including hop-limited forwarding, map-based geographic forwarding, and timer-based simple forwarding [15]. In the hop-limited forwarding, a predetermined number of hops are specified for a message to be propagated. Yet in map-based forwarding, messages are flowed based on geographical areas. The last solution operates at the network layer and aims to maximize accessibility in highly disconnected networks [16]. The proposed solutions aim to achieve a high message delivery ratio with minimum contention and collision.

Using relaying protocols, a source vehicle can select the nearest or furthest node (i.e., relay node) within its vicinity. Only few vehicles are chosen for the broadcasting process which guarantees network scalability. Several protocols concerning relay node selection have been proposed. The first trial was Urban Multi-hop Broadcast (UMB) protocol that divides the transmission range into segments and a relay node is chosen from the furthest segment [15]. The UMB protocol uses $\mathrm{RTB} / \mathrm{CTB}$ handshaking mechanism and repeater infrastructures at the intersection. Afterward, a modified version of UMB is introduced in [17] known as Ad-hoc Multi-hop Broadcast (AMB) protocol. This protocol eliminates the usage of repeaters and instead it selects the closest vehicle to that intersection (as shown Fig. 2). 


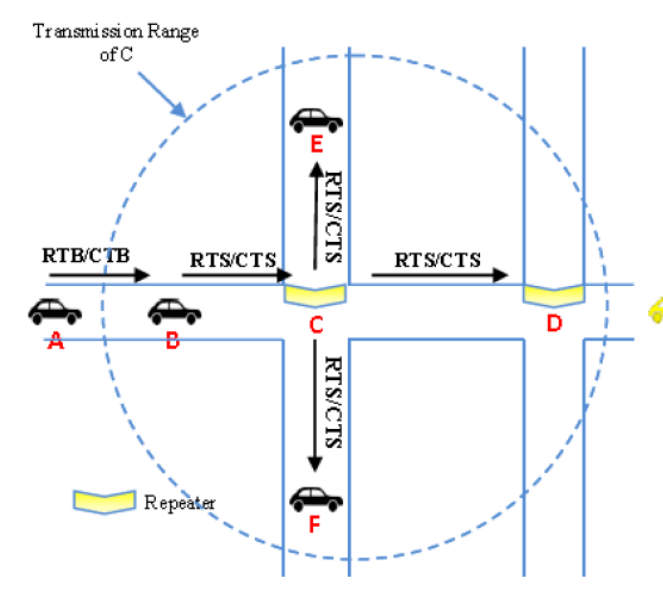

(a) UMB protocol

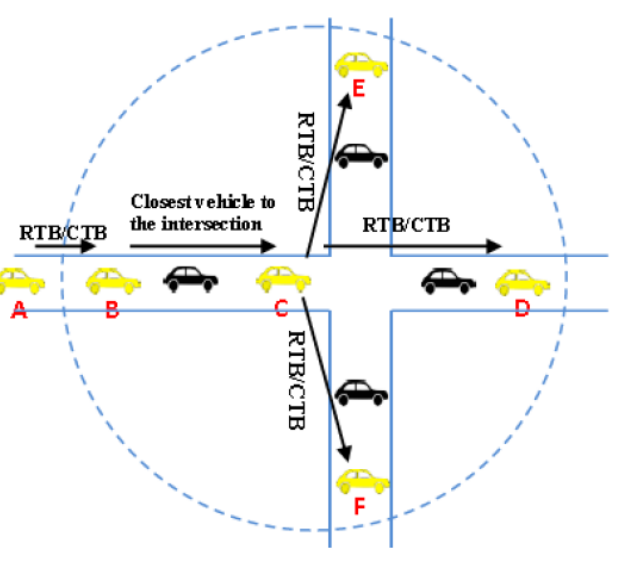

(b) AMB protocol

Fig. 2 Difference between AMB and UMB protocols

Smart Broadcast (SB) is presented in [18] in which the transmission range of the source node is also divided into segments. Unlike UMB and AMB protocols, it assigns a small contention window to the furthest segment instead of using jamming signal. Due to the drawbacks of using a static contention window, a time-based segmentation protocol is proposed [19] and [20]. In this protocol, the relay node is selected from the nearest segment in order to first transmit the safety message to vehicles within the danger zone.

Vehicle-to-Roadside Protocols. Mitigate vehicle crashes, broadcast traffic messages, and provide entertainment data are popular benefits of using V2R communications. However, an RSU cannot occasionally provide the needed services, as a result a remote sites accessing are mandatory. According to MQTT protocol [9], the RSU is a broker between vehicles (i.e., subscribers) and service providers (i.e., publishers). Publisher/Subscriber messaging systems prevent the direct communication between vehicles and service providers. This MQTT protocol is essential especially for networks suffering from high latency.

Due to the vast applications offered by RSUs, MD protocols are categorized into two approaches which are pull-based [21] and push-based approach [22]. Using the pull-based approach, vehicles can request information from an RSU. It is suitable for user-specific message delivery however it may cause contentions and collisions. On the contrary is the second approach where RSUs periodically broadcast beacons to all vehicles within its transmission range. Push-based approach is used in applications related to unexpected events, or accidents causing safety hazards. The main goal of this approach is to disseminate messages including parameters such as location, direction, and speed. In V2R communication, the pull-based approach is more consistent, reliable and scalable compared to the push-based approach.

\section{Existing Simulation Tools for VANETs}

Examining network performance in real life seems to be impossible due to huge expenses and network complexity. Hence, using simulation tools is the best mean to obtain real-world vehicular scenarios. In Fig. 3, network simulation tools are classified into three categories. Nevertheless, simulating VANETs protocols require including wireless technologies such as IEEE 802.11p [6], and IEEE 1609 [23]. 


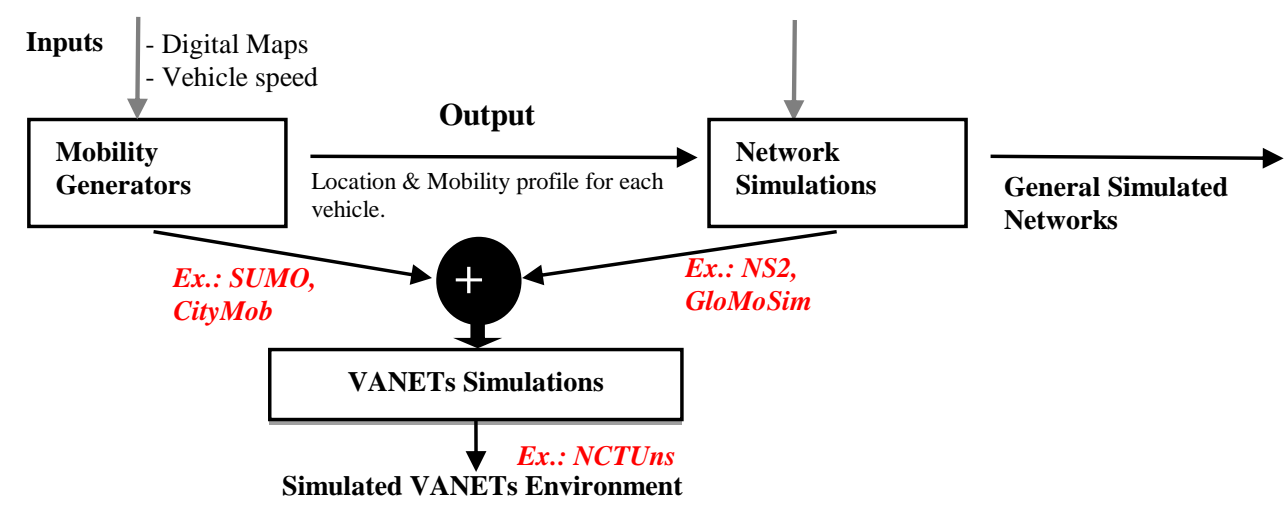

Fig. 3 Relationship among Network Simulation Tools

Networks Simulators. Unlike MANETs, it is not possible in most cases to simulate VANETs with using network simulators. VANETs extensions are required (i.e. mobility generators) to be able to mimic real vehicular environments. Network simulators are suitable to test novel protocols, or apply modifications to existing ones. Common simulators include Network Simulator2 (NS2) [24], Global Mobile Information System simulator (GloMoSim) [25] and Scalable Wireless Ad Hoc Network Simulator (SWANS) [26].

NS2 is a discrete and object-oriented tool developed by University of California at Berkeley and VINT project [24]. It supports the simulation of TCP and routing protocols over both wired and wireless networks. Object Tool Command Language (OTcl) is used to enable objects to be compiled in $\mathrm{C}++$. The second popular simulator comes after NS2 is the GloMoSim [25]. This simulator uses a C-like programming language called Parsec with the ability to divide its workload into separate modules to reduce the CPU load. On the other hand, SWANS [26] a general-purpose simulator based on Java in Simulation Time (JiST) engine [27]. It has a unique feature over other network simulators in which it can run standard Java applications such as peer-to-peer applications. We summarize the main features of the mentioned network simulators in Table 2.

Table 2 Main features of ns-2 and Glomosim

\begin{tabular}{|c|c|c|c|c|}
\hline & & NS-2 & GloMoSim & SWANS \\
\hline \multirow{4}{*}{$\begin{array}{l}\text { Software } \\
\text { Properties }\end{array}$} & -Commons & \multicolumn{3}{|c|}{ Portable, freeware, open source, console, GUI } \\
\hline & -Setup & \multirow{3}{*}{$\begin{array}{c}\text { Easy } \\
\text { High } \\
\text { OTcl scripts in } \\
\text { ClC++ }\end{array}$} & \multirow{3}{*}{$\begin{array}{c}\text { Moderate } \\
\text { low } \\
\text { Parsec }\end{array}$} & \multirow{3}{*}{$\begin{array}{c}\text { Easy } \\
\text { low } \\
\text { Java }\end{array}$} \\
\hline & -CPU\&Memory & & & \\
\hline & -Language used & & & \\
\hline \multirow{2}{*}{$\begin{array}{c}\text { Concerning } \\
\text { VANETs }\end{array}$} & -IEEE $802.11 p$ & \multirow{2}{*}{$\begin{array}{l}\text { Yes } \\
\text { No } \\
\end{array}$} & \multirow{2}{*}{$\begin{array}{l}\text { No } \\
\text { No }\end{array}$} & \multirow{2}{*}{$\begin{array}{l}\text { Yes } \\
\text { Yes } \\
\end{array}$} \\
\hline & -Traffic model & & & \\
\hline \multirow[b]{2}{*}{ Features } & -Advantages & $\begin{array}{l}\text { large scripts and } \\
\text { Split-language } \\
\text { programming }\end{array}$ & $\begin{array}{l}\text { Run on } \\
\text { concurrent } \\
\text { memory }\end{array}$ & $\begin{array}{l}\text { Save memory and } \\
\text { run Java } \\
\text { application }\end{array}$ \\
\hline & -Disadvantages & $\begin{array}{l}\text { Manual } \\
\text { programming and } \\
\text { Only Bi- and } \\
\text { Omni- directional } \\
\text { antennas }\end{array}$ & $\begin{array}{l}\text { No Traffic } \\
\text { models and } \\
\text { obstacles. }\end{array}$ & $\begin{array}{l}\text { Environmental } \\
\text { models are not } \\
\text { sufficient as in } \\
\text { NS2 }\end{array}$ \\
\hline
\end{tabular}

Mobility Generator. Mimic VANETs requires simulating mobility traces which are achieved by using mobility generators. They generate a detailed profile for each vehicle besides the road models to be used as inputs for network simulators. In this subsection, we present a brief description for some mobility generators and list their criteria in Table 3. Simulation of Urban Mobility (SUMO) is a microscopic open-source generator that manages different network formats [28]. It is a highly 
portable generator which makes it suitable for different network simulators. VANET MobiSim is another popular mobility generators based on Java language [29]. The generated trace file of VANET MobiSim can be obtained using Google Earth or TIGER databases. These files are then sent to the network simulators without returning any feedback. For the CityMob generator, it can implement a downtown model and adjust vehicles speed according to how dense is the simulated area [30]. It is compatible with NS-3 network simulator.

Table 3 Available mobilty generators and simulator

\begin{tabular}{|c|c|c|c|c|c|}
\hline & & SUMO & MOVE & $\begin{array}{l}\text { VANET } \\
\text { MobiSim }\end{array}$ & CityMob \\
\hline \multirow{3}{*}{$\begin{array}{l}\text { Software } \\
\text { Properties }\end{array}$} & -Features & \multicolumn{4}{|c|}{ User-friendly GUI, open source, and freeware software } \\
\hline & -Setup & Moderate & Easy & Moderate & Easy \\
\hline & -Usage & Hard & Moderate & Moderate & Easy \\
\hline \multirow{2}{*}{ Maps used } & -Real maps & Yes & Yes & Yes & No \\
\hline & -User-defined & Yes & Yes & Yes & No \\
\hline \multirow{2}{*}{$\begin{array}{l}\text { Mobility } \\
\text { Models }\end{array}$} & -Features & \multicolumn{4}{|c|}{$\begin{array}{l}\text { Random waypoint, multilane roads, lane changing, } \\
\text { directional flow, and traffic signs }\end{array}$} \\
\hline & -Route calculation & Yes & Yes & Yes & No \\
\hline \multirow{4}{*}{$\begin{array}{c}\text { Traces } \\
\text { supported }\end{array}$} & -NS-2 trace & No & Yes & Yes & Yes \\
\hline & -GloMoSim & No & Yes & Yes & No \\
\hline & -XML trace & No & No & Yes & No \\
\hline & -Different formats & Yes & Yes & Yes & No \\
\hline
\end{tabular}

VANETs Simulators. VANET simulators are integrated environments that allow researchers to implement different applications related to VANETs. As in Table 4, we present NCTUns and GrooveNet as an example for VANET simulators. NCTUns is an abbreviation for National Chia Tung University Network Simulator [31]. Although it is built using C++ language, it has many unique features that make it less complex compared to NS2. Due to the novelty of NCTUns, it is an open-source simulator that faces some drawbacks which are: 1) lack of tutorials especially dealing with errors, 2) using Fedora 9 Linux as a platform, and 3) limitation of the maximum number of nodes supported (i.e., 4096 nodes). Another VANET simulator is the GrooveNET, mainly developed for vehicular environment, which support several message types such as GPS messages or vehicle emergency messages [32]. GrooveNET can generate real-street maps for any place in United States of America by importing TIGER files from the US Census Bureau. This simulator outperforms NCTUns for being easier to setup and use.

Table 4 A comparison between NCTUns and groovent

\begin{tabular}{|c|c|c|c|}
\hline & & NCTUns & GrooveNet \\
\hline \multirow{3}{*}{$\begin{array}{l}\text { Integrated } \\
\text { Framework }\end{array}$} & -Features & General network purposes & Vehicular Environments \\
\hline & -Mobility Models & Random and manual routes & $\begin{array}{ll}\text { Random } & \text { and } \\
\text { origin-destination } & \\
\end{array}$ \\
\hline & -Setup & Hard & Moderate \\
\hline \multirow[t]{3}{*}{ Traffic Model } & -Speed models & Random & Uniform \\
\hline & -Road topology & User-defined & Any \\
\hline & - Traffic lights & Automatically & Manually defined \\
\hline VANETs & - IEEE $802.11 \mathrm{p}$ & \multicolumn{2}{|c|}{ Both can support this standard } \\
\hline $\begin{array}{l}\text { protocols } \\
\text { facilities }\end{array}$ & -Built-in applications & None & $\begin{array}{l}\text { Vehicle warning and } \\
\text { rebroadcasting }\end{array}$ \\
\hline
\end{tabular}

\section{Conclusions}

The increasing popularity of VANETs has attracted many researchers to compete for developing robust MD protocols. In this paper, we focus on VANETs main characteristics compared to MANETs, 
system components and design requirements of VANETs. Additionally, we elaborate the current delivery protocols. Methods of information delivery and the types of the needed information have been mentioned. Nonetheless, future work is required to handle the content information in terms of usage, sharing and security. Finally, we go through the popular simulation tools related to VANETs. They are categorized into mobility generators, network simulators and VANETs simulators. We mentioned the most popular commercial tools and we hold a comparison concerning the major features of simulation software in each category.

\section{Acknowledgements}

This work was supported by National Key Technology R\&D Program (2012BAD35B06), The National High-Tech Research and Development Plan of China under Grant No.2012AA01A301-0 and The National Natural Science Foundation of China (61173036).

\section{References}

[1] S. An, B.H. Lee and D.R. Shin, in: A Survey of Intelligent Transportation Systems, 2011 Third International Conference on Computational Intelligence, Communication Systems and Networks (CICSyN), Bali (2011), p. 332-337.

[2] F. Li and Y. Wang, in: Routing in Vehicular Ad Hoc Networks: A Survey, Volume 2 in IEEE Vehicular Technology Magazine, Number 2, (2007), p. 12-22.

[3] W. Chen, R.K. Guha, T.J. Kwon and J. Lee, in: A Survey and Challenges in Routing and Data Dissemination in Vehicular Ad Hoc Networks, Volume 11 in Wireless Communications and Mobile Computing, Number 7, (2011), p. 787-795.

[4] H. Kawashima, in: Japanese Perspective of Driver Information Systems, Volume 17 in Transportation, Number 3, (1990), p. 263-284.

[5] Y. Li, Y. Yu and S. Li, in: A Survey on Unicast Routing Protocol for Vehicular Ad Hoc Networks, in: Advances in Electrical Engineering and Automation, Berlin (2012), p. 253-258.

[6] Y.J. Li, in: An Overview of the DSRC/WAVE Technology, in Quality, Reliability, Security and Robustness in Heterogeneous Networks, Berlin (2012), p. 544-558.

[7] S. Olariu and M.C. Weigle: Vehicular Networks: From Theory to Practice (Crc Press, United States 2009).

[8] C. Lochert, B. Scheuermann, C. Wewetzer, A. Luebke and M. Mauve, in: Data Aggregation and Roadside Unit Placement for a Vanet Traffic Information System, Proceedings of the fifth ACM international workshop on VehiculAr Inter-NETworking, San Francisco (2008), p. 58-65.

[9] U. Hunkeler, H.L. Truong and A. Stanford-Clark, in: MQTT-S-A Publish/Subscribe Protocol for Wireless Sensor Networks, 3rd International Conference on communication Systems Software and Middleware and Workshops (COMSWARE), Bangalore (2008), p. 791-798.

[10] P.T. Eugster, P.A. Felber, R. Guerraoui, and A.M. Kermarrec, in: The Many Faces of Publish/Subscribe, Volume 35 in ACM Computing Surveys (CSUR), Number 2, (2003), p. 114-131.

[11] Q. Xu, T. Mak, J. Ko and R. Sengupta, in: Vehicle-To-Vehicle Safety Messaging in DSRC, Proceedings of the 1st ACM international workshop on Vehicular ad hoc networks, Philadelphia (2004), p. 19-28. 
[12] C.L. Robinson, L. Caminiti, D. Caveney and K. Laberteaux, in: Efficient Coordination and Transmission of Data for Cooperative Vehicular Safety Applications, Proceedings of the 3rd international workshop on Vehicular ad hoc networks, Los Angeles (2006), p. 10-19.

[13] Information on http://www.travelmidwest.com/lmiga/home.jsp.

[14] P. Salvo, M. De Felice, F. Cuomo and A. Baiocchi, in: Infotainment Traffic Flow Dissemination in an Urban VANET, IEEE Global Communications Conference (GLOBECOM), Anaheim (2012), p. 67-72.

[15] G. Korkmaz, E. Ekici, F. Özgüner and Ü. Özgüner, in: Urban Multi-Hop Broadcast Protocol for Inter-Vehicle Communication Systems, Proceedings of the 1st ACM international workshop on Vehicular ad hoc networks, Philadelphia (2004), p. 76-85.

[16] L. Briesemeister, L. Schafers and G.Hommel, in: Disseminating Messages Among Highly Mobile Hosts Based on Inter-Vehicle Communication, Proceedings of the IEEE Intelligent Vehicles Symposium IV 2000, Dearborn (2000), p. 522-527.

[17] G. Korkmaz, E. Ekici and F. Ozguner, in: An Efficient Fully Ad-Hoc Multi-Hop Broadcast Protocol for Inter-Vehicular Communication Systems, IEEE International Conference on Communications (ICC'06), Istanbul (2006), p. 423-428.

[18] E. Fasolo, A. Zanella and M. Zorzi, in: An Effective Broadcast Scheme for Alert Message Propagation in Vehicular Ad Hoc Networks, IEEE International Conference on Communications (ICC'06), Istanbul (2006), p. 3960-3965.

[19] M.M. Taha, Y.M. Hasan, in: VANET-DSRC Protocol for Reliable Broadcasting of Life Safety Messages, IEEE International Symposium on Signal Processing and Information Technology, Cairo (2007), p. 104-109.

[20] M.M. Taha, Y.M. Hasan, in: A Novel Headway-Based Vehicle-to-Vehicle Multi-Mode Broadcasting Protocol, IEEE 68th Vehicular Technology Conference (VTC 2008-Fall), Calgary (2008), p. 1-5.

[21] V. Kumar and N. Chand, in: Data Scheduling in Vanets: A Review, Volume 1 in International Journal of Computer Science and Communication, Number 2, (2010), p. 399-403.

[22] P. Tomar, B.K. Chaurasia and G.S. Tomar, in: State of the Art of Data Dissemination in Vanets, Volume 2 in International Journal of Computer Theory and Engineering, Number 6, (2010), p. 1793-8201.

[23] I.T.S Committee, in: IEEE Trial-Use Standard for Wireless Access in Vehicular Environments (WAVE)—Networking Services, IEEE Std: 1609-3, (2007).

[24] K. Fall and K. Varadhan, in: Ns Notes and Documents, The VINT Project - LBL, USC/ISI, and Xerox PARC 705, Berkeley (2000).

[25] X. Zeng, R. Bagrodia and M. Gerla, in: GLOMOSIM: A Library for Parallel Simulation of Large-Scale Wireless Networks, Proceedings of the twelfth workshop on Parallel and distributed simulation (PADS 98), Banff (1998), p. 154-161.

[26] J. Wu: Handbook on Theoretical and Algorithmic Aspects of Sensor, Ad Hoc Wireless, and Peer-to-Peer Networks (Crc Press, United States 2005).

[27] R. Barr, Z.J. Haas and R. van Renesse, in: JIST: An Efficient Approach to Simulation Using Virtual Machines, Volume 35 in Software: Practice and Experience, Number 6, (2005), p. 539-576. 
[28] D. Krajzewicz, G. Hertkorn, C. Rössel and P. Wagner, in: Sumo (Simulation of Urban Mobility), Proceedings of the 4th Middle East Symposium on Simulation and Modelling, Sharjah (2002), p. 183-187.

[29] J. Härri, F. Filali, C. Bonnet and M. Fiore, in: VanetMobiSim: Generating Realistic Mobility Patterns for VANETS, Proceedings of the 3rd international workshop on Vehicular ad hoc networks(ACM), Los Angeles (2006), p. 96-97.

[30] F.J. Martinez, J.C. Cano, C.T. Calafate and P. Manzoni, in: Citymob: A Mobility Model Pattern Generator for VANETs, IEEE International Conference on Communications Workshops (ICC Workshops' 08), Beijing (2008), p. 370-374.

[31] Information on http://ns110.csie.nctu.edu.tw/.

[32] R. Mangharam, D. Weller, R. Rajkumar, P. Mudalige and F. Bai, in: Groovenet: A Hybrid Simulator for Vehicle-to-Vehicle Networks, The 3rd Annual International Conference on Mobile and Ubiquitous Systems: Networks and Services (MOBIQUITOUS), San Jose (2006), p. 1-8. 